OPEN ACCESS

Edited by:

Jose J. Munoz,

Universitat Politecnica de Catalunya,

Spain

Reviewed by:

Fabio Giavazzi,

University of Milan, Italy

José Rafael Bordin,

Federal University of Pelotas, Brazil

Jennifer A. Mitchel,

Harvard University, United States

*Correspondence:

Josef $A$. Käs

jkaes@uni-leipzig.de

Specialty section:

This article was submitted to

Biophysics,

a section of the journal

Frontiers in Physics

Received: 10 February 2021

Accepted: 24 May 2021

Published: 17 August 2021

Citation:

Blauth E, Kubitschke H, Gottheil P, Grosser S and Käs JA (2021) Jamming

in Embryogenesis and

Cancer Progression.

Front. Phys. 9:666709.

doi: 10.3389/fphy.2021.666709

\section{Jamming in Embryogenesis and Cancer Progression}

\author{
Eliane Blauth, Hans Kubitschke, Pablo Gottheil, Steffen Grosser and Josef A. Käs * \\ Peter-Debye Institute for Soft Matter Physics, Leipzig University, Leipzig, Germany
}

The ability of tissues and cells to move and rearrange is central to a broad range of diverse biological processes such as tissue remodeling and rearrangement in embryogenesis, cell migration in wound healing, or cancer progression. These processes are linked to a solidlike to fluid-like transition, also known as unjamming transition, a not rigorously defined framework that describes switching between a stable, resting state and an active, moving state. Various mechanisms, that is, proliferation and motility, are critical drivers for the (un) jamming transition on the cellular scale. However, beyond the scope of these fundamental mechanisms of cells, a unifying understanding remains to be established. During embryogenesis, the proliferation rate of cells is high, and the number density is continuously increasing, which indicates number-density-driven jamming. In contrast, cells have to unjam in tissues that are already densely packed during tumor progression, pointing toward a shape-driven unjamming transition. Here, we review recent investigations of jamming transitions during embryogenesis and cancer progression and pursue the question of how they might be interlinked. We discuss the role of density and shape during the jamming transition and the different biological factors driving it.

Keywords: embryogenesis, cancer, jamming, unjamming, jamming transition, physics of cancer, morphogenesis, glass transition

\section{INTRODUCTION}

Spatiotemporal tissue organization is crucial in various biological processes ranging from fundamental shaping of tissues during embryogenesis, tissue fluidization during wound healing, and pathological alterations in diseases like asthma [1-3] and cancer [4-6]. Sculpting biological tissues is a demanding task, from both an (epi)genetic and physical perspective. Typically, there is no external force driving tissue deformation and cellular rearrangement. Instead, the organisms and tissues generate internal forces themselves. A significant ingredient for reshaping tissues is to locally control the flux of cells while remaining in a global structurally stable state. All cells and proto-tissues are in a fluid, viscous regime; however, larger tissues require further mechanical integrity, which a fluid cannot provide.

A solid-like tissue with force and tension percolation can exert forces onto a fluid-like without compromising its structural integrity by the counter-acting forces. Conversely, fluid-like tissues can deform and shear, enabling cells to moving along or through other tissues. Losing cell-cell contacts to one cell and establishing new contacts to new cells is another primary ingredient in reshaping tissues. Without this switching of cellular neighbors, the tissue cannot fundamentally reshape and restructure.

In recent years, observations and theoretical descriptions of sudden fluidization, collective motion arrest, and solidification of tissues elucidated how tissues organize and structure. Local control of viscoelasticity, rigidity, and viscosity is necessary for shaping tissues during embryogenesis [7-10]. On the other hand, it also harbors the risk of failure when tissue does not comply with its intended 
viscoelastic properties, for example, diseases like cancer. The current concept toward understanding viscoelastic changes in tissues in the scientific community is that tissues can undergo a rigidity transition, similar to a phase transition. In fluids and solids, physical properties change rapidly at the transition point. Also, sudden changes in viscoelastic properties are seen in biological tissues during embryogenesis and cancer progression.

The leading theoretical framework describing rigidity transitions and sudden collective motion arrest in biological systems is the theory of a jamming transition. Despite the jamming transition is being well-defined in the condensed physics literature [11-13], the biological physics community, however, uses Jamming and Jamming Transition sometimes a rather descriptive phenomenon for an observed rigidification, solidification, or motion arrest in tissues. Unjamming and jamming transitions are currently interpreted as driving principles for dynamical tissue organization and mechanical integrity [3-6, 10, 14-19]. Here, biological cells are seen as rather densely packed, viscoelastic cells with additional properties, depending on the exact theoretical model, such as motility, adhesion, and cortical tension, as mediators for force generation and tension percolation.

Nonetheless, connecting theory and experimental observation and thus unraveling the fundamental cause of the rigidity transition in biological tissues is still a challenging task [3, 5, 20, 21]. For one, jamming and unjamming and similar theories in biological tissues made significant theoretical advances. Nonetheless, accessible experimental data is sparse; pinpointing exact causes and driving factors is complex.

In this review, we will briefly outline different theoretical interpretations of the jamming phenomena in biological tissues. We cover principles of the rigidity transition of biological systems and discuss recent findings in theory and experiment and disputed views. A key determinant in jamming debated in the scientific community is whether jamming is cell-shape- or cell-density-driven. We focus on embryogenesis and cancer progression and discuss both interpretations from experimental observations. We support jamming as a universal principle in embryogenesis and cancer, albeit the driving factors in both classes differ. We hypothesize that embryogenesis favors density-driven whereas cancer progression favors the cell-shape-induced interpretation of the jamming phenomena. We will outline open questions and investigation into the theory of jamming in biological systems in this review. For some of those, we provide mindful and tentative answers and hypotheses as an incentive for future research. As the accessible experimental data is sparse, we elucidate possible future experiments and possible contributions to deepen our understanding of the jamming phenomenon.

\section{SHORT INTRODUCTION TO CELL JAMMING}

\subsection{Jamming in Colloidal Systems}

In passive colloidal or grainy systems, jamming is the transition ranging between gas-, liquid-, solid-, and liquid crystalline-like states controlled by density and temperature [22]. With increasing density, particle motion is more and more constrained by its neighbors.
When these systems reach a critical density $\rho_{c}$, single-particle motion becomes caged, and the systems become solid-like. However, it is still a matter of debate whether this transition is an actual phase transition.

In the context of this review, it is decisive to understand that the critical parameter in colloid jamming is the particle density $\rho$ or their volume fraction $\phi$. The exact value of the critical parameter depends on the dimensionality of the system. Moreover, it can be modulated by external stresses $\sigma$. For instance, roundish colloids in three dimensions jam at a volume fraction of around $\phi=64 \%$ [23].

Remarkably, the particle shape can shift the critical parameter in colloid systems. Elongated particles need higher volume fractions to jam than roundish particles [24]-elongated particles have more degrees of freedom and need more neighbors to be constrained. In a colloid system, particle shape is usually simply given; shape-dependent effects play somewhat less of a decisive role. However, cells have highly adaptable shapes, which is one of the main reasons cell shape potentially serves as a control parameter in dense cell systems.

\subsection{Jamming in Biological Systems: Density and Shape}

Like colloidal systems, the extracellular space and thus the density can control the transition from an unjammed to a jammed state [10]. More extracellular space results in more uncorrelated cellular movement and fluidizes the system. Increasing the density jams the system, and cellular movement becomes confined by neighboring cells $[5,10]$.

A striking feature of cell systems is that they can considerably tune their number density at high volume fractions close to $100 \%$. The cell number can increase due to proliferation or external pressure. An increase in number density is accompanied by a glass-like slowing down of cell motility in 2D monolayers [14, 25]. Similar behavior was even found in $3 \mathrm{D}$ cell aggregates $[4,26]$. At first sight, it seems that density is the main driving factor in these systems. However, the question of how cells move at such high volume fractions close to one remains. Hard sphere colloidal jamming happens at much lower volume fractions, for example, $\phi=0.74$ is the densest packing ratio for hard spheres; however, random packing of spheres will already jam at approx. $\phi=0.64$ [23]. One key feature of cellular systems is their ability to adapt their shapes. Cells are viscoelastic objects and can deform and actively generate stresses to reshape themselves on the timescale of minutes. Thus, even though they can form confluent layers or 3D cell aggregates with a volume fraction close to 1 , they might overcome the jamming constraints of hard spheres by elongating, thereby extending their degrees of freedom.

The concept of shape-dependent unjamming has been strengthened by many experimental studies in the last few years. The slowing-down of cellular motion was associated with more roundish cell shapes in a broad range of systems from $2 \mathrm{D}$ monolayers to $3 \mathrm{D}$ cell aggregates and primary tumor pieces [1, 3-5, 25, 27, 28]. In densely packed environments, unlike in typical colloidal systems, cells can still move if they elongate and squeeze themselves through narrow spaces (Figure 1). 
A

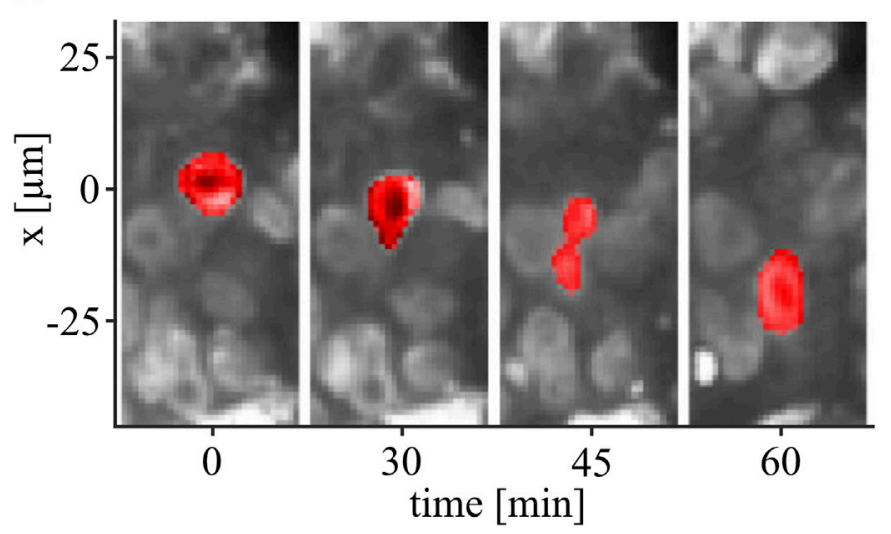

B

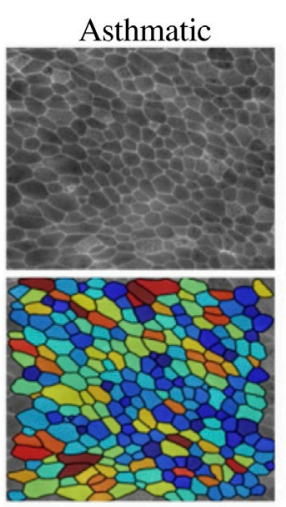

Non-asthmatic

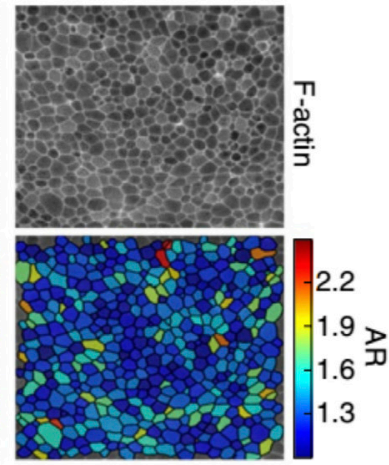

FIGURE 1 | Shape-dependent unjamming. (A) Time series of an MDA-MB-436 cancer cell nucleus (red) deforming itself to move through a densely packed threedimensional cell spheroid. Nuclei are stained with SiR-DNA. From [4](B), monolayers of cells from asthmatic donors were more motile and had more elongated cells than monolayers of cells from healthy donors. Images were taken and adopted from Atia et al. [28]. Copyright ID 5061921078636.

Atia et al. have found that shape elongation is tied to unjamming across a broad range of biological systems [28]. They empirically find that the shape distributions in biological systems are linked to k-Gamma-distributions found in granular materials. The shape variability increases as the system unjams and decreases when the system approaches the jamming point. This indicates that the jamming transition in living systems is also driven by geometrical constraints similar to inert systems [28].

All of the mentioned studies point toward a significant role of the cell shape as a geometric constraint, thus reducing the system's degrees of freedom leading to jamming transitions. As cells are complex living systems, cellular properties influence the cell shape and thus control the transition.

\subsection{The SPV Model as the Paradigmatic Model of Shape-Dependent Jamming}

Besides these experimental studies, theoretical models were developed to describe the cell jamming transition. The most influential models are arguably the vertex- and the selfpropelled Voronoi (SPV) model, as they predict a densityindependent and shape-dependent transition. For this reason, we want to outline the crucial features of both models. The main difference between both models is the way that they obtain the cell shapes. In the vertex model, the tissue is described by a polygonal tiling of space. The Hamiltonian depends on the vertex positions, which correspond to the degrees of freedom. On the other hand, in the SPV model, cell shapes are given by a Voronoi tessellation around the cell positions. Thus, the SPV model has fewer degrees of freedom. Nonetheless, it comes to similar conclusions. Its Hamiltonian is given by the following equation:

$$
E=\sum_{i=1}^{N} E_{i}=\sum_{i=1}^{N}\left[K_{A}\left(A\left(r_{i}\right)-A_{0}\right)^{2}+K_{P}\left(P\left(r_{i}\right)-P_{0}\right)^{2}\right] .
$$

Here, $A_{i}$ and $P_{i}$ are the area and perimeter of each cell, their respective moduli $K_{A, i}$ and $K_{P, i}$, and the (theoretical) target values $A_{0}$ and $P_{0}$. The models assume that the energetically favored perimeter $P_{0}$ reflects the competition between cortical tension and cell-cell adhesion, and the target area $A_{0}$ accounts for the monolayer's resistance to height fluctuations. In this view, strong cell-cell adhesions and low cortical tension elongate the cells, and the system is driven toward unjamming. In the SPV model, cells can be additionally actively self-propelled. For vanishing selfpropulsion, the model predicts a jamming transition for cells with a target shape index $p^{*}=\frac{P_{0}^{*}}{\sqrt{A_{0}^{*}}}=3.81$, which is similar to the vertex model [20].

Increased cellular self-propulsion, which suggests stronger traction forces, pushes the target shape index for the onset of jamming toward more roundish shapes [20]. The prediction of a collective cell motility arrest at a cell shape index of around $p^{*}=$ 3.81 matched the experimental observation in monolayers [3]. Since then, the model has been refined and adapted by introducing, for example, heterogeneity of cell divisions [29, 30]. Chaing et al. also found a shape-dependent transition in a Potts model [31]. We do not want to discuss these in detail as the SPV model serves as a paradigm that explains the mechanism of shape-dependent transitions in dense tissues.

\section{JAMMING IN EMBRYOGENESIS}

Particularly during the early stages of embryogenesis, tissues are continuously reshaped and restructured, while cells constantly proliferate and differentiate within a single day [32]. Physical cues are guiding morphogenetic rearrangements of tissues with high spatiotemporal precision [33-37]. One key ingredient is local solidification and fluidization of tissue.

In early seminal works, Foty and Steinberg $[38,39]$ formulated the differential adhesion hypothesis explaining the demixing of different cell types during embryogenesis without relying on intricate biological cues and function. Instead, tissues are treated as liquids with different surface tensions and arrange according to their surface tension, like oil droplets in water. The hypothesis of differential adhesion provided an excellent initial 


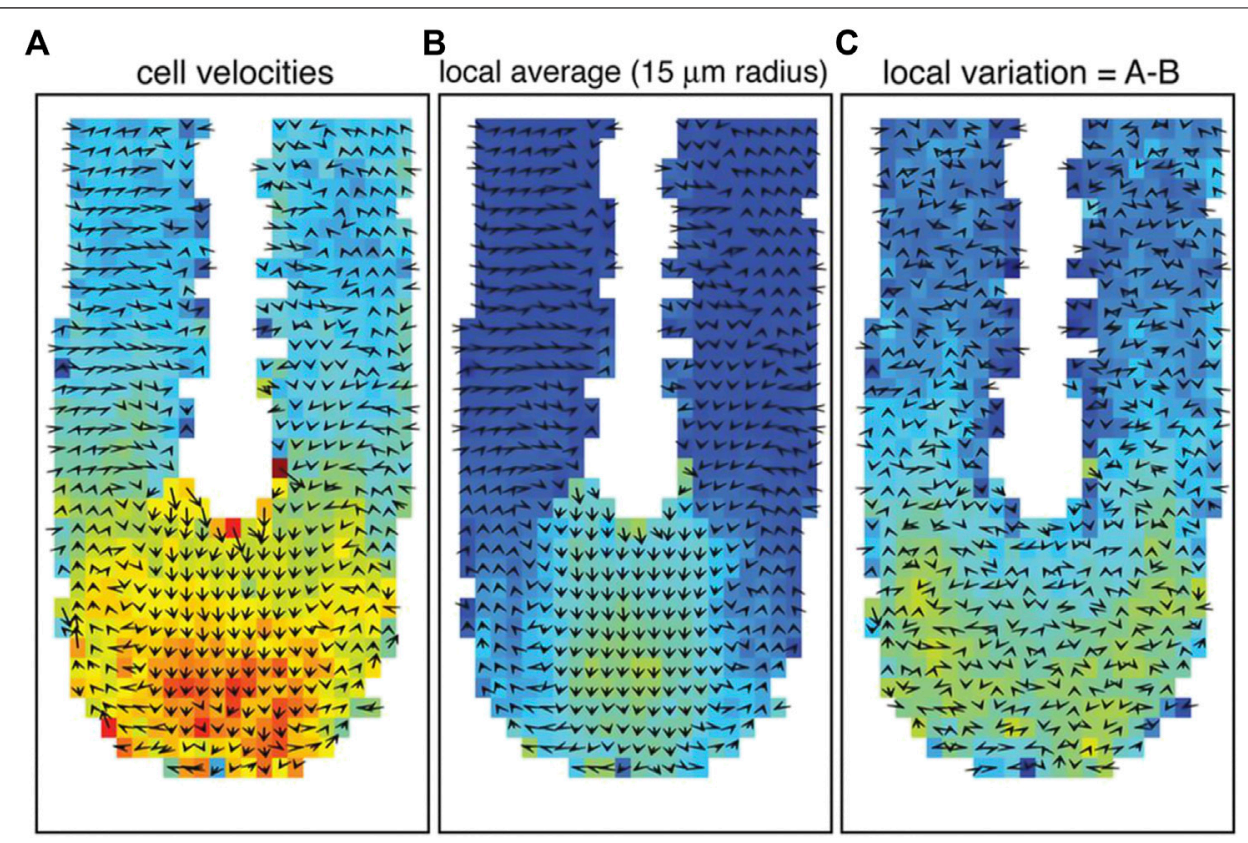

FIGURE 2 | Cell movement and tissue fluidity in the zebrafish tailbud. (A) Vector map of cell velocities. (B) Local tissue velocity averaged over a 15 um radius. (C) Cell velocity variation from local tissue velocity. The color red represents higher velocities, and arrows indicate the averaged velocity vectors in $2 \mathrm{D}$. The tip of the tailbud displays higher cell velocities, more local rearrangements, and proliferation, indicating an unjammed, fluid-like state. High motility and proliferation are driving factors for unjamming tissues, as verified by Mongera et al. [10]. Images were taken and adopted from Lawton et al. [48], Copyright ID 1096693-1.

explanation of physical guiding principles during embryogenesis [38-43]. However, cellular adhesion strength is not sufficient to fully describe physical actions in embryogenesis and tissue development $[44,45]$. In this context, jamming and unjamming were proposed as processes influencing the demixing of cells [45]. Like the differential adhesion hypothesis, the jamming phenomenon is also based on adhesion forces, cell density, and shape.

The vertebrate body axis elongation is an essential step in forming the anteroposterior body axis and a hallmark of animal development [46]. The physical mechanisms that control this process are still not fully understood; however, a study by Mongera et al. showed that it might be driven by localized, mesoscopic jamming and unjamming. During the formation of the vertebrate body axis in zebrafish, the posterior tissue solidifies at the presomitic mesoderm (PSM) while proliferating at the still fluid-like mesodermal progenitor zone (MPZ) [10].

This observation matches previous studies showing a different mode of cell movement during the development of the vertebrate axis $[47,48]$. More specifically, the cells in the posterior tip move randomly and individually or fluid-like. At the same time, they become slower and move collectively or instead solidify in the PSM (see Figure 2). This corresponds precisely to a jamming transition at the tip of the vertebrate body axis [48].

In this process, the jamming transition is spatiotemporally controlled by the extracellular space regulated by cadherindependent cell-cell adhesions [10, 48]. However, it remains unclear whether jamming transitions during embryogenesis are generally density-driven. The solidification along the vertebrate body axis results in unidirectional growth and elongation of the embryo. Supracellular stresses act as mechanical cues for the morphogenetic flow.

Similar to the localized proliferation and (un)jamming during the vertebrate body elongation, tissue jamming and unjamming are also present during branching morphogenesis. In this process, tubular structures are formed from remodeling epithelial or endothelial sheaths, resulting in branched structures such as ducts of the kidney, mammary and salivary glands, or lung tissue [49-51].

Other embryonic processes in which jamming transitions play a role are the ventral furrow formation in Drosophila and the early stages of gastrulation [7, 28]. This short overview shows how spatiotemporal control of fluid-like and solid-like tissue states by jamming is essential during tissue morphogenesis.

\section{UNJAMMING IN CANCER}

In contrast to embryogenesis, cell rearrangements within healthy adult tissues happen only rarely, for example, during wound healing. Still, in some diseases, cells regain the ability to migrate, for instance, metastatic cancer cells. Carcinomas are tumors that develop from epithelial cells. The healthy epithelium separates and protects different organs from each other. It needs to be stable, organized, and mechanically intact against external forces. From a biophysical point of view, bounding epithelium can be seen as a tissue that is in a jammed state. During cancer progression, the cancer cells start to proliferate in an uncontrolled way, grow to a solid tumor, and even migrate through the body. The transition from the stable non-motile 

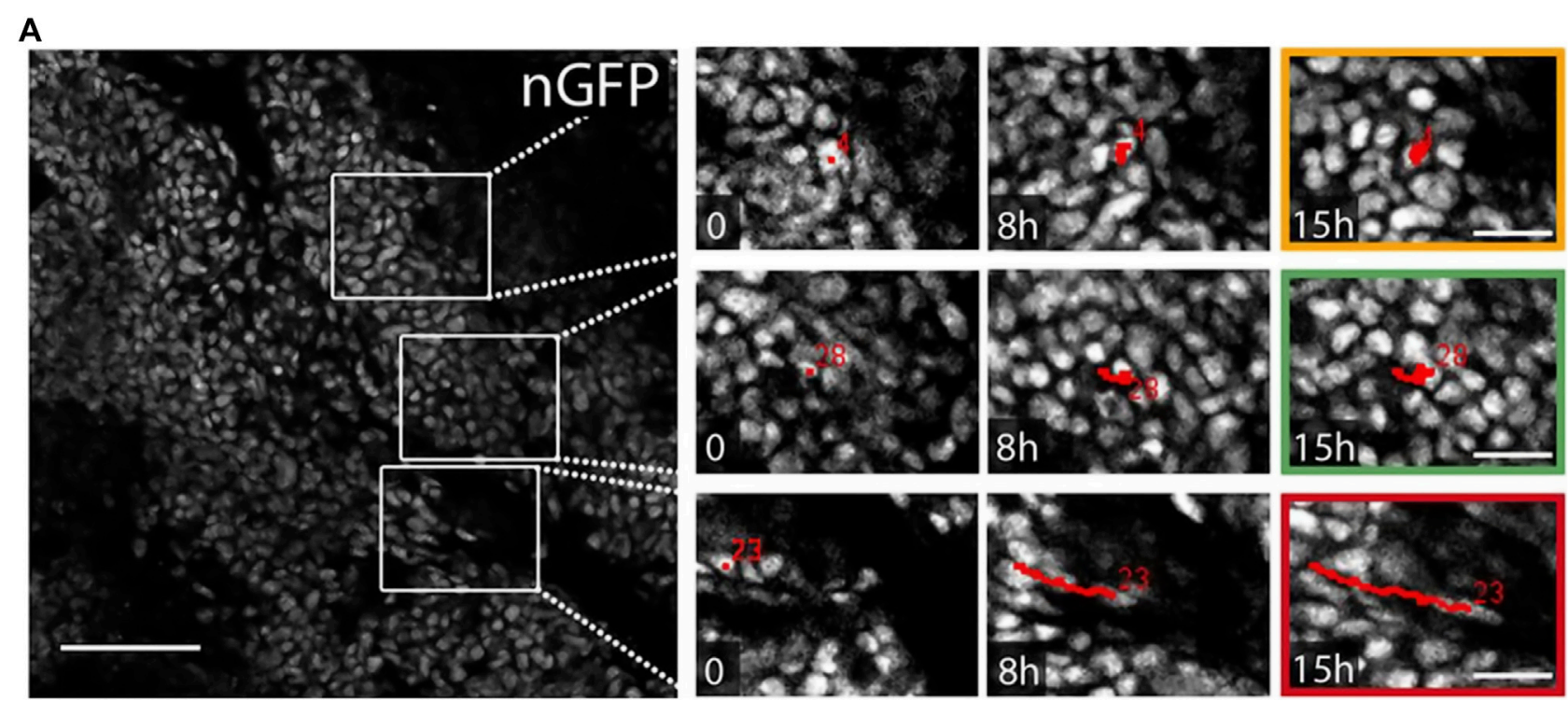

FIGURE 3 | Tumors have jammed and unjammed regions. Cell migration tracks in the core of tumor explants from mice. The ex vivo tumor shows jammed regions with resting cells (yellow box) and unjammed regions with moving cells (green and red box). The unjammed areas display different migration dynamics, with high (red) or lower (green) persistence. Red numbers represent the track ID numbers. Nuclei are stained with nuclear-targeting GFP (nGFP). Scale bar 100 mm. Images were taken and adapted from Staneva et al. [55], Copyright ID 1096785-1.

epithelium to an unstructured, partly motile tumor can thus be seen as an unjamming transition.

The idea to link the migration of cancer cells to unjamming has already been proposed some years ago [6]. Particularly, the link between the EMT and unjamming seemed natural because, during EMT, cells detach from the solid tumor and migrate outwards, which resembles the fluidization of the tissue. However, it is still under debate if EMT and unjamming are similar processes. In a 2D model system, inducing partial EMT lead to different structural and dynamical signatures than a pressure-induced unjamming transition [1]. However, deregulation of adherens junctions and downregulation of E-cadherin in epithelial tissues, which is a crucial step during EMT, has been shown to drive invading cells toward a more unjammed state [5]. The biological processes of cancer invasion are heterogeneous, and the cells can adopt different migration modes. The physical concept of unjamming represents a unifying framework that is agnostic of molecular details.

This seems to hold for the pure tumor as well as for the compound of tumor and microenvironment. While the presence of moving cells at the invasive tumor front seems intuitive-most tumors are composed of a proliferation outer rim of cancer cells [52-54] - unjamming appears in the tumor core as well. Recent studies found fluid-like regions with correlated moving cells in tumor explants from humans and mice $[4,55]$. This observation is represented in Figure 3. Here, the tracks of cancer cells in tumor explants point toward a wide variety of dynamic behavior inside densely packed tumors. This raises the question of the role of jamming and unjamming in the overall tumor stability and shape. Even though uncontrolled proliferation is a hallmark of cancer, both reports conclude that proliferation is not the driving factor for the unjamming transition $[4,55]$.
Tumors often grow in close vicinity to connective and fatty tissues [56]. While only little is known about the influence of fatty tissue on the unjamming of cancer cells, connective tissue, mainly composed of ECM, has been shown to influence the dynamical behavior of cancer cells. A dilute ECM network can induce a transition to a gas-like state, where cells move individually. Denser networks result in constrained and collective motion and can even block cellular motion $[5,57]$.

In cancer progression, a crucial determinant in patient survival rate is the occurrence of metastasis. Metastasizing cells can be hypothesized as single unjammed cells escaping a jammed tumorous tissue. The heterogeneous vertex model predicts that single unjammed cells are relatively unlikely, and jamming is a (localized) collective property [30]. For instance, a mixture of one cell type that typically jams and another that stays unjammed would form a globally jammed layer when the mixing ratios of jammed and unjammed cells reach $4: 1$, thereby trapping the "fluid" unjammed cells in a scaffold of "solid" jammed cells [30,58]. The encasement of fluid cells by jammed cells results in the percolation of the tension network, which significantly increases the rigidity of the tissue [30]. In essence, soft cells can still form rigid tissues via tension percolation and jamming, thus explaining why rigid tumors contain soft cells [6].

Particularly in the tumor cores, the question of how cells can rearrange in an already densely packed environment, near volume fraction 1, arises. In contrast to embryogenesis, where changes in density have a major role in the dynamical behavior of the cells, the cell shape is a critical parameter in dense tissues. It can be used as a marker for tissue fluidity [4]. In clinical cancer grading, the variance of nuclear shapes and sizes-so-called pleomorphisms [59]-is used as a crucial marker for the 
aggressiveness of tumors. The nuclear shape further correlates with the cell shape [4]. A higher grading, which implies more heterogeneous nuclear shapes, means that, statistically, the cell shapes have a higher variability if the correlation is valid for primary tumors. Since cancer cell clusters are amorphous aggregates, higher variances are accompanied by higher average values [28]. Thus, pleomorphism, that is, high variance in nuclear shapes, might be accompanied by higher cell and nuclear shapes in tumors [4, 28]. This circumstance yields a contextual link between conventional clinical cancer grading and the physics of unjamming.

\section{CURRENT PROBLEMS IN CELL JAMMING}

\subsection{Driving Factors of the Unjamming Transition}

In real biological systems and models, the number density, the volume fraction, and the cell shapes play a role in the jamming transition. However, cell shapes and densities are not intrinsic properties of cells but result from an interplay of cell-cell adhesion, substrate adhesion, cell activity (such as traction generation or contractile stress), and cell stiffness. Particularly in cancer progression, it is essential to understand which factors shape cells in a dense environment and support migration. From a physical point of view, one can ask, what are the different axes of the jamming phase diagram?

An emerging key feature of more unjammed cell monolayers is strong cell-substrate interaction combined with high traction forces. This has been shown throughout many studies in the last years [3, 25, 60, 61]. Some studies have argued that high substrate traction implies strong cell-cell adhesion to balance and transmit the high intercellular tensile stresses [3, 60, 61]. However, a recent study explicitly tried to disentangle the contributions of cell-cell adhesion and substrate traction, pointing out that the adhesion strength does not change visibly in a more unjammed monolayer [25]. Saraswathibathla et al. even demonstrated that stress fiber alignment and the related traction forces predominantly control the cell shape and, finally, the unjamming transition [25]. A possible explanation for the dominant role of traction forces could be that they are stronger than the forces at the cellular interfaces. Thus, their influence on the cell shapes prevails over cell-cell adhesion and cortical tension effects, which are important control parameters in the SPV model.

In confluent systems without any cell-substrate interaction, for example, three-dimensional cell spheroids, this substrate effect should vanish, and the role of the cell-cell adhesion becomes dominant. The predictions of the 3D-SPV model [62], however, were not confirmed by an experimental study [4]. In fact, cell spheroids built from less adhesive cells were more unjammed, and single cells could move through the spheroid (Figure 1), while the more adhesive cells formed jammed spheroids with non-motile cells [4].

In another study that tested the influence of cell-cell adhesion on the dynamics, the authors investigated a non-confluent system by placing cell clusters in a substrate and ECM environment. The strength of cell-cell adhesion clearly influenced cell behavior. Here, a decrease of cell-cell adhesion leads to more individualized and faster cell movement, more elongated cells, and fluidizes the system further (see Figure 4) [5]. Additionally, the weaker cellcell contacts allowed the system to promote unjamming again through a density decrease [5].

In both studies, the authors clearly find an association between unjamming and cell shapes $[4,5]$, but these were not associated with higher cell-cell adhesion as suggested by the SPV [16, 62].

The question of whether cell-cell adhesion or substrate traction drives the unjamming transition can thus not be clearly answered in general. The role of cell-cell adhesion seems to be more complex than predicted by the SPV model. Some experimental evidence showed that weak adhesion supports unjamming and promotes invasive cell behavior. This behavior fits with the known role of EMT in cancer progression.

\subsection{Nuclear Jamming}

Cell jamming theories have consistently emphasized cell shapes rather than nucleus shapes because the whole cells are volume-filling

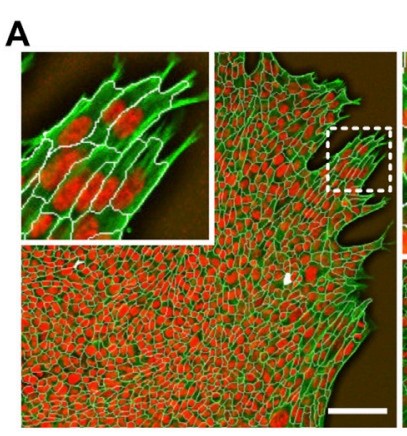

shNT

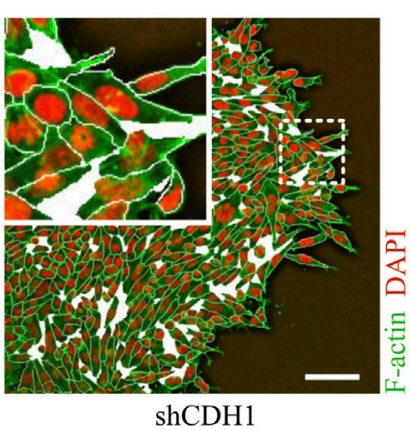

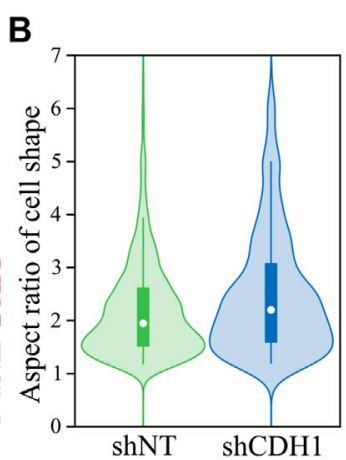

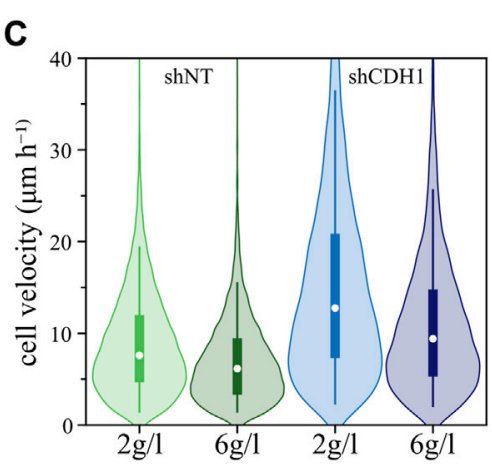

FIGURE 4 | Downregulation of E-cadherin promotes unjamming in migrating cell layer. (A) Morphology of wild-type (shNT) and E-cadherin downregulated (shCDH1) cell layers migrating at a collagen interface. The wild-type cell aggregate is almost area-filling and exhibits high spatial correlations for the long axes-the orientation-of the cells. E-Cadherin knockout cell aggregate displays density fluctuations, and cells are randomly aligned. Actin is stained in green (Alexa Fluor 568-conjugated phalloidin), while nuclei are stained in red (DAPI). The white lines visualize a cell segmentation with holes in the tissue represented by white areas. The scale bar represents $100 \mu \mathrm{m}$. (B) Change of aspect ratio due to loss of E-cadherin. An increase in aspect ratio corresponds to higher shape indices, thus promoting unjamming of tissue. (C) Change in cell migration velocity due to downregulated E-cadherin and promoted unjamming. Graphics and data were adopted from llina et al. [5]. 
a tissue - not the nuclei - which is a crucial element in various theories $[3,16,28]$. In other words, cells in dense tissues should be jammed, while nuclei alone would not be jammed, as their volume fraction is much lower. Recent studies, however, suggest that the nucleus might actively be involved.

The role of the nucleus in single-cell migration has been clear for quite a while. The ability of the cell nuclei to squeeze into free spaces was found to be essential for tumor progression [63, 64]. While the cytoplasm of migrating cells can move through pore sizes below $1 \mu \mathrm{m}$, this is not the case for the nucleus as it is the stiffest cell organelle [65-67]. The nuclear compressibility and size further limit the single-cell movement through narrow spaces [68-71]. However, the nucleus not only has a limiting effect on cell migration in dense environments. Two recent studies showed that the nucleus itself is mechanosensitive and that nuclear deformation enhances cellular force generation [72, 73]. Moreover, Staneva et al. and Grosser et al. demonstrated that cell nuclei in tumor clusters strongly deform while they move through the tissue (Figure 1) [4, 55]. Unjammed regions in tumor samples were characterized by elongated nuclei [4], typically found in elongated cells.

From single-cell movement to primary cancer samples, these observations suggest that the nucleus is actively and passively involved in jamming, potentially adding to the traditional role of nuclei shapes in cancer.

\subsection{Motion Arrest or Rigidity Transition}

In cell systems, the terms jamming and unjamming are often used loosely. By jamming, some authors refer to collective motion arrest $[1,3,74]$ or locally caged behavior [25, 58]. Others call the collective motility arrest glass transition, avoiding the term jamming [14], or simply rigidity transition [16]. In fact, cellular systems exhibit many features typical of glasses, such as an amorphous, disordered structure, a broad range of relaxation time scales, and a flat plateau region of the mean squared displacements on intermediate time scales [14, 20, 61, 75].

A key feature of jamming is that a system that jams actually becomes solid-like. However, the connection between motion arrest and rigidity is not obvious for non-equilibrium systems such as tissues. Tissues are active matter, and various biological and physical mechanisms are intertwined and work simultaneously. They potentially react actively to external stress, for example, by fluidization. Motion, or lack thereof, cannot sufficiently determine jamming and unjamming in tissues in the sense of a true rigidity transition. Fluid-like systems are characterized by stress relaxation, whereas solid-like systems typically show no or only minor stress relaxation. So far, only Serwane and Mongera et al. have provided stress relaxation data in vertebrate body axis formation and connected the data to the jamming phenomenon $[10,76]$.

An epithelial sheet that moves into a cell-free area could be in a motile but (locally) jammed state. Their neighbors cage cells within the sheet while the whole sheet collectively moves [58, 77]. Conversely, tissues could be in a non-rigid, low tension, unjammed state with only minimal or vacant cell motion insufficient for active neighbor exchange [78]. The relation between rigidity and jamming is of particular interest in cancer development. Recent research has suggested that unjamming is involved in cancer progression. However, tumors are usually

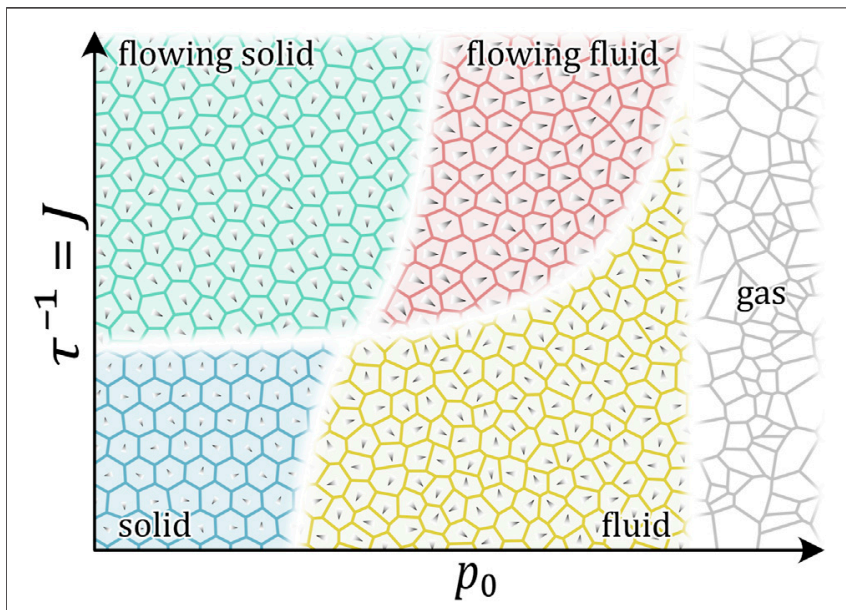

FIGURE 5 | Qualitative phase diagram of 2D cell monolayers at volume fraction close to 1 . The phase diagram is based on [61, 75]. Control parameters are the target shape index $p_{0}$ and the alignment interaction $\mathrm{J}=\tau^{-1}$ with $\tau$ being the reorientation time which is the time that cells take to adjust their polarities along the direction of the velocities of the corresponding local environments. The magnitude of the motility $v_{0}$ and its direction is indicated as arrows. Tissue approaches hexagonal-like structure for decreasing target shape factor. With increasing alignment interaction, the system displays more long-ranged directed motility. Blue cells in phase space: alignment interactions are minor, and cellular reorientations are governed by rotational diffusion. The target shape index is small yielding roundish cells. Here, cells rarely align with neighborhood velocities and are randomly oriented. The energetically favored shape index is low-yielding hexagonal-like structures. Green cells in phase space: roundish cells and hexagonal-like tissue structures are present induced by a small target shape index. Highvelocity correlations yield flowing flocks of solid-like cell aggregates. Red cells in phase space: high-velocity correlations accompanied by elongated cells yield a fluid phase in which aggregates undergo coordinated motion. Yellow cells in phase space: moderate to vanishing alignment interactions and elongated cells yield a fluid phase where cells undergo predominantly random motion. Gray cells in phase space: cell interactions vanish in this phase due to the high target shape index. The cellular system behaves gas-like.

harder than their surroundings. Future research has still to connect the dots between hard tumors, soft cells [79], and the role of unjamming in cancer [4-6].

There might be a rather non-physical explanation why the term jamming has become popular. It is because it intuitively expresses the collective, emergent character of this transition-"clearly, jamming is not something that you can do on your own" [80].

\subsection{Different Migration Modes of Unjamming}

In the literature, the word unjamming is used for various dynamical behavior in biological systems. The observed migration patterns range from the collective motion of sheets or packs [1, 14, 61, 74, 81] to single-cell squeezing events $[4,25]$, or T1-transitions [82]. These different migration modes can be distinguished by respective characteristic velocity correlation length, migration persistence, or pack size of cooperatively migrating cell clusters.

Cells modify their polarization and migration direction through interaction with surrounding cells [18, 83], which might lead to more collective motion [18, 83]. Theoretical 

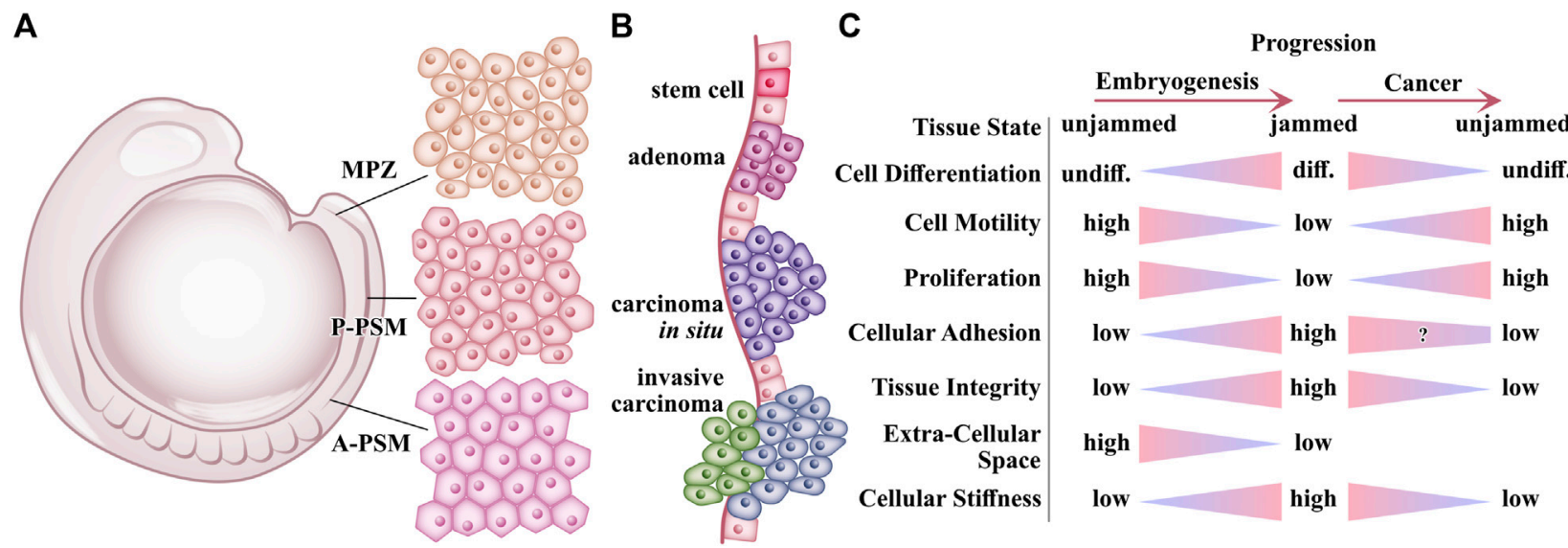

FIGURE 6 | Occurrence of Jamming and unjamming in embryogenesis and cancer. (A) Illustration of the lateral view of zebrafish embryo in the 10-somite stage. During periodic rapid elongations at the tail of the embryo, cells from the mesodermal progenitor zone (MPZ) transform to cells of the posterior (P-PSM) presomitic mesoderm and consecutively to anterior presomitic mesoderm (A-PSM). During this transformation, the initial cells of the MPZ slowly decrease the cell motility, filling the voids of the extracellular space, and restructuring their $\mathrm{N}$-cadherin-mediated adhesion with neighboring cells resulting in increased tissue stiffness and integrity [10]. (B) Cancer progression during early stages. Growing factors like TGF- $\beta$ limit the growth of normal epithelium and early-stage tumors resulting in adenomas. After losing growth-inhibitory responsiveness, tumors grow more rapidly, transforming into carcinomas in situ. Additional oncogenic mutations are acquired during rapid growth. By chance, tumor cells with specific oncogenic mutations can undergo epithelial-mesenchymal transdifferentiation in response to TGF- $\beta$ and are becoming more invasive carcinomas [90]. (C) Overview of changes on tissue and cellular level during embryogenesis and cancer progression. Various processes and properties which change during embryogenesis are reversed during cancer progression, motivating the hypothesis of cancer being inverse morphogenesis [87, 88, 91].

models take this into account by introducing an additional alignment interaction. The resulting jamming phase diagram contains four distinct phases: solid/jammed, solid flock/flowing solid/active nematics, liquid flock/flowing liquid, and liquid (see Figure 5) [61, 75]. These states represent the experimentally observed migration patterns in more detail and show that unjamming can mean different types of motion. This further raises the question of whether the dynamical signature of moving cells can give information about the interaction strength of cells?

This further raises the question of whether the dynamical signature of moving cells can provide information about the interaction strength of cells.

The observed migration modes also resemble the ones seen in active nematic fluids. Self-propelled rods with a higher aspect ratio, similar to elongated mesenchymal cells, display phases of flocking, laning, and isotropic alignment with sharp phase transitions. Furthermore, the flocking motion in active nematics, although collectively moving, is internally, locally jammed [84], which can be observed in biological tissues as well $[4,10]$. It should be further noticed that cellular systems might also show mixed jammed/unjammed stated as it is, for example, seen in spheroids embedded in collagen, where the core can be still jammed while the outer shell starts to melt $[26,74]$.

\section{SHAPE- AND DENSITY-DRIVEN JAMMING IN EMBRYOGENESIS AND CANCER}

In recent years, it has become clear that rigidity transitions are involved in embryogenesis and diseases. While early studies and modeling approaches had focused on $2 \mathrm{D}$ in vitro monolayers and 2D models [3, 14, 20, 21], crucial breakthroughs were made by several studies that observed the jamming phenomenon in $3 \mathrm{D}$ systems $[4,5,10,55,74]$. These studies comprise in vitro but also ex vivo and in vivo systems.

Early experiments pointed to different, even opposing, roles in these processes (see Figure 6). Experimentally, jamminglike fluid-to-solid transition accompanied embryogenesis [10, 47, 48, 85]. During embryonic development, cells proliferate quickly and rearrange while they differentiate into specialized cells and form stable, structured organs. The cellular stiffness increases with differentiation [86]. Eventually, proliferation and migration subside, suggesting increasing solidification in the course of embryogenesis. The tissues are in a jammedlike state.

During cancer progression, by contrast, cells dedifferentiate. For instance, in cervical cancer, cancer cells de-differentiate and spread from the tissue of origin to tissues of ontogenetic proximity in reverse order to the mature derivatives of the morphogenetic fields [87, 88]. The cancer cell stiffness declines with increasing malignancy [79, 89]. Metastatic cells lose cell-cell adhesion and gain higher motility. This results in a decline in tissue integrity under further tumor growth and cancer cell invasion. These trends suggest a central role of unjamming and fluidization in cancer. Several recent studies found this effect in cancer systems $[4,5$, $61,74]$. In short, many processes in embryogenesis are reversed in cancer progression, including jamming and unjamming.

Originally, the term jamming was chosen in analogy to colloidal systems that rigidify with increasing density. Cell 
number density in epithelial monolayers was the first driver of jamming to be reported [14]. However, observations in an asthma model have suggested cell shape as the central switch between jamming and unjamming [3]. Consequently, the SPV models $[16,20,62]$ have become an inspiring driver of research that promoted a new physics: cell-shape-dependent jamming. Several years later, both shape-dependent jamming and density-dependent jamming have experimentally been found $[3-5,10,28]$.

However, one can only hypothesize the potentially different roles these two mechanisms play based on the still limited evidence. Rapidly increasing cell density and the maturation and stabilization of cell-cell contacts characterized embryogenesis, solidifying the system. Experimentally, the shrinking volume of extracellular space and increasing adhesion drive jamming during zebrafish development [10] and other systems [7], in line with the global tendencies during embryogenesis. However, shape also plays a role during embryogenesis [28], especially considering 2D-like tissues such as epithelial sheets. Cancer progression and the formation of metastases show an opposing tendency: cancer starts in a dense environment. Tumors grow within an already existing organism against outside homeostatic pressure, resulting in an effective volume constraint. Thus, if cancer progression is accompanied by unjamming, the global environment suggests a more prominent shape-induced unjamming in tumors. In recent years, shape-induced unjamming was found, with several recent studies pointing to shape-dependent unjamming in tumor clusters [4] as well as complex tumor-ECM environments [5] and even asthma [3].

\section{CONCLUSION AND OUTLOOK}

Observed in embryogenesis and diseases, tissues continuously alter their rigidity and reshape. Solidification of tissues drives embryogenesis, whereas fluidization of cancer cells drives cancer progression, leading to metastasis. Recent findings support the concept of a jamming-like transition as a concept of tissue remodeling of rigidity and fluidity modulation. With this review, we want to shed light on the plurivalent concepts of the jamming phenomenon. While tissue (un)jamming can be induced by different intracellular (E-cadherin, RAB5A, stress fiber alignment) $[4,5,25,61,74]$ and extracellular mechanisms such as pressure $[3,28]$, density $[5,10]$, irradiation [27], or ECM confinements [5], it has turned out to be a remarkable overarching principle that unites processes stemming from a wide variety of cues. Nonetheless, which physical quantity or parameter is the driving factor for jamming and unjamming is still debatable. Given the complexity of the biological matter, it is most likely that more than one parameter should be considered in jamming transitions. Experimental data of embryonic tissues favors cell-density whereas cancer models prefer a shape-induced explanation of the jamming phenomenon. The jamming phenomenon has likely more than one or two sides.
While theoretical models made large advancements in recent years, the experimental data needed for verification or falsification is incomplete. The biological and physical mechanisms of tissues have to be carefully dissected. Reliable quantification of jamming-related quantities is a challenging task.

Future experiments under controlled conditions should further elucidate how stresses in jammed and unjammed tissues relax. These experiments also provide the opportunity to identify (and perhaps quantify) more molecular details involved in stress relaxation in tissue. Cell-cell adhesion generates tension in tissues. Thus, density-driven cell crowding can significantly generate rigidity in tissue as predicted in vertex and SPV models $[62,92]$. When the cell density increases, the number, and thus strength of cell-cell contacts increases. At a critical density, sufficient force bridges are generated, the tension network percolates and the tissue undergoes a rigidity transition [30, 93]. The rigidity percolation can also encase fluid-like cells by rigid cells, which significantly increases the rigidity of the tissue [30]. Data of local tissue rigidity current provides the most convincing link to the jamming phenomenon $[10,93]$ and supports the interpretation of jamming as a density-driven transition as seen in embryogenesis. As the largest and stiffest organelle, the cell nucleus harbors significant potential for the observed rigidity percolation. In models (and experiments), the feedback (loop) between nuclear deformation and cell migration/force generation is not sufficiently investigated. The nucleus and its importance in jamming were only considered in one experiment by Grosser et al. [4]. Cells, however, are mechanosensitive and adapt to the rigidity of their environment [94-96]. Thus, experiments addressing rigidity will have difficulties in dissecting physical aspects from biological aspects. On the other hand, these difficulties are also a large opportunity to link jamming to the molecular origin.

\section{AUTHOR CONTRIBUTIONS}

All authors discussed the results and contributed to the final manuscript; provided critical feedback and helped shape the manuscript; and contributed to writing the manuscript.

\section{FUNDING}

This project has received funding from the European Research Council (ERC-741350/HoldCancerBack). We acknowledge funding from the European Commission H2020-PHC-2015two-stage as part of the 'FORCE' project (668039/Imaging the Force of Cancer). We acknowledge support from the German Research Foundation (DFG) and University of Leipzig within the program of Open Access Publishing.

\section{ACKNOWLEDGMENTS}

We like to thank Jürgen Lippoldt for fruitful discussions and vital feedback for this manuscript. 


\section{REFERENCES}

1. Mitchel JA, Das A, O’Sullivan MJ, Stancil IT, DeCamp SJ, Koehler S, et al. In Primary Airway Epithelial Cells, the Unjamming Transition Is Distinct from the Epithelial-To-Mesenchymal Transition. Nat Commun (2020) 11:1-14. doi:10.1038/s41467-020-18841-7

2. Park JA, Atia L, Mitchel JA, Fredberg JJ, and Butler JP. Collective Migration and Cell Jamming in Asthma, Cancer and Development. J Cel Sci (2016) 129: 3375-83. doi:10.1242/jcs.187922

3. Park JA, Kim JH, Bi D, Mitchel JA, Qazvini NT, Tantisira K, et al. Unjamming and Cell Shape in the Asthmatic Airway Epithelium. Nat Mater (2015) 14: 1040-8. doi:10.1038/nmat4357

4. Grosser S, Lippoldt J, Oswald L, Merkel M, Sussman DM, Renner F, et al. Cell and Nucleus Shape as an Indicator of Tissue Fluidity in Carcinoma. Phys Rev X (2021) 11:011033. doi:10.1103/PhysRevX.11.011033

5. Ilina O, Gritsenko PG, Syga S, Lippoldt J, La Porta CAM, Chepizhko O, et al. Cell-cell Adhesion and 3D Matrix Confinement Determine Jamming Transitions in Breast Cancer Invasion. Nat Cel Biol (2020) 22:1103-15. doi:10.1038/s41556-020-0552-6

6. Oswald L, Grosser S, Smith DM, and Käs JA. Jamming Transitions in Cancer. J Phys D: Appl Phys (2017) 50:483001. doi:10.1088/1361-6463/aa8e83

7. Petridou NI, Grigolon S, Salbreux G, Hannezo E, and Heisenberg CP. Fluidization-mediated Tissue Spreading by Mitotic Cell Rounding and Non-canonical Wnt Signalling. Nat Cel Biol (2019) 21:169-78. doi:10.1038/ s41556-018-0247-4

8. Petridou NI, and Heisenberg CP. Tissue Rheology in Embryonic Organization. EMBO J (2019) 38:e102497. doi:10.15252/embj.2019102497

9. Barriga EH, and Mayor R. Adjustable Viscoelasticity Allows for Efficient Collective Cell Migration. Semin Cel Dev Biol (2019) 93:55-68. doi:10.1016/ j.semcdb.2018.05.027

10. Mongera A, Rowghanian P, Gustafson HJ, Shelton E, Kealhofer DA, Carn EK, et al. A Fluid-To-Solid Jamming Transition Underlies Vertebrate Body axis Elongation. Nature (2018) 561:401-5. doi:10.1038/s41586-0180479-2

11. Berthier L, Flenner E, and Szamel G. Glassy Dynamics in Dense Systems of Active Particles. J Chem Phys (2019) 150:200901.

12. Liu AJ, and Nagel SR. The Jamming Transition and the Marginally Jammed Solid. Annu Rev Condens Matter Phys (2010) 1:347-69. doi:10.1146/annurevconmatphys-070909-104045

13. Charbonneau P, Kurchan J, Parisi G, Urbani P, and Zamponi F. Glass and Jamming Transitions: From Exact Results to Finite-Dimensional Descriptions. Annu Rev Condens Matter Phys (2017) 8:265-88. doi:10.1146/annurevconmatphys-031016-025334

14. Angelini TE, Hannezo E, Trepat X, Marquez M, Fredberg JJ, and Weitz DA. Glass-like Dynamics of Collective Cell Migration. Proc Natl Acad Sci U.S.A (2011) 108:4714-9. doi:10.1073/pnas.1010059108

15. Bi D, Lopez JH, Schwarz JM, and Manning ML. Energy Barriers and Cell Migration in Densely Packed Tissues. Soft Matter (2014) 10:1885-90. doi:10.1039/C3SM52893F

16. Bi D, Lopez JH, Schwarz JM, and Manning ML. A Density-independent Rigidity Transition in Biological Tissues. Nat Phys (2015) 11:1074-9. doi:10.1038/nphys3471

17. Farhadifar R, Röper JC, Aigouy B, Eaton S, and Jülicher F. The Influence of Cell Mechanics, Cell-Cell Interactions, and Proliferation on Epithelial Packing. Curr Biol (2007) 17:2095-104. doi:10.1016/j.cub.2007.11.049

18. Sadati M, Taheri Qazvini N, Krishnan R, Park CY, and Fredberg JJ. Collective Migration and Cell Jamming. Differentiation (2013) 86:121-5. doi:10.1016/ j.diff.2013.02.005

19. Schötz EM, Lanio M, Talbot JA, and Manning ML. Glassy Dynamics in ThreeDimensional Embryonic Tissues. J R Soc Interf (2013) 10. doi:10.1098/ rsif.2013.0726

20. Bi D, Yang X, Marchetti MC, and Manning ML. Motility-Driven Glass and Jamming Transitions in Biological Tissues. Phys Rev X (2016) 6:021011. doi:10.1103/PhysRevX.6.021011

21. Garcia S, Hannezo E, Elgeti J, Joanny JF, Silberzan P, and Gov NS. Physics of Active Jamming during Collective Cellular Motion in a Monolayer. Proc Natl Acad Sci (2015) 112:15314-9. doi:10.1073/pnas.1510973112
22. Anderson VJ, and Lekkerkerker HNW. Insights into Phase Transition Kinetics from Colloid Science. Nature (2002) 416:811-5. doi:10.1038/416811a

23. Song C, Wang P, and Makse HA. A Phase Diagram for Jammed Matter. Nature (2008) 453:629-32. doi:10.1038/nature06981

24. Donev A, Cisse I, Sachs D, Variano EA, Stillinger FH, Connelly R, et al. Improving the Density of Jammed Disordered Packings Using Ellipsoids. Science (2004) 303:990-3. doi:10.1126/science.1093010

25. Saraswathibhatla A, and Notbohm J. Tractions and Stress Fibers Control Cell Shape and Rearrangements in Collective Cell Migration. Phys Rev X (2020) 10: 011016. doi:10.1103/PhysRevX.10.011016

26. Han YL, Pegoraro AF, Li H, Li K, Yuan Y, Xu G, et al. Cell Swelling, Softening and Invasion in a Three-Dimensional Breast Cancer Model. Nat Phys (2020) 16:101-8. doi:10.1038/s41567-019-0680-8

27. O’Sullivan MJ, Mitchel JA, Das A, Koehler S, Levine H, Bi D, et al. Irradiation Induces Epithelial Cell Unjamming. Front Cel Dev Biol (2020) 8. doi:10.3389/ fcell.2020.00021

28. Atia L, Bi D, Sharma Y, Mitchel JA, Gweon B, Koehler A, et al. Geometric Constraints during Epithelial Jamming. Nat Phys (2018) 14:613-20. doi:10.1038/s41567-018-0089-9

29. Czajkowski M, Sussman DM, Marchetti MC, and Manning ML. Glassy Dynamics in Models of Confluent Tissue with Mitosis and Apoptosis. Soft Matter (2019) 15:9133-49. doi:10.1039/C9SM00916G

30. Li X, Das A, and Bi D. Mechanical Heterogeneity in Tissues Promotes Rigidity and Controls Cellular Invasion. Phys Rev Lett (2019) 123:058101. doi:10.1103/ PhysRevLett.123.058101

31. Chiang M, and Marenduzzo D. Glass Transitions in the Cellular Potts Model. EPL (2016) 116:28009. doi:10.1209/0295-5075/116/28009

32. Briggs JA, Weinreb C, Wagner DE, Megason S, Peshkin L, Kirschner MW, et al. The Dynamics of Gene Expression in Vertebrate Embryogenesis at Single-Cell Resolution. Science (2018) 360. doi:10.1126/science.aar5780

33. Keller R, Davidson LA, and Shook DR. How We Are Shaped: The Biomechanics of Gastrulation. Differentiation (2003) 71:171-205. doi:10.1046/j.1432-0436.2003.710301.x

34. Adamo L, Naveiras O, Wenzel PL, McKinney-Freeman S, Mack PJ, GraciaSancho J, et al. Biomechanical Forces Promote Embryonic Haematopoiesis. Nature (2009) 459:1131-5. doi:10.1038/nature08073

35. Responte DJ, Lee JK, Hu JC, and Athanasiou KA. Biomechanics-driven Chondrogenesis: from Embryo to Adult. FASEB J (2012) 26:3614. doi:10.1096/fj.12-207241

36. Delile J, Herrmann M, Peyriéras N, and Doursat R. A Cell-Based Computational Model of Early Embryogenesis Coupling Mechanical Behaviour and Gene Regulation. Nat Commun (2017) 8:1-10. doi:10.1038/ ncomms13929

37. Heisenberg CP, and Bellaïche Y. Forces in Tissue Morphogenesis and Patterning. Cell (2013) 153:948-62. doi:10.1016/j.cell.2013.05.008

38. Foty RA, Pfleger CM, Forgacs G, and Steinberg MS. Surface Tensions of Embryonic Tissues Predict Their Mutual Envelopment Behavior. Development (1996) 122:1611-20.

39. Steinberg MS. ON THE MECHANISM OF TISSUE RECONSTRUCTION BY DISSOCIATED CELLS, III. FREE ENERGY RELATIONS AND THE REORGANIZATION OF FUSED, HETERONOMIC TISSUE FRAGMENTS. Proc Natl Acad Sci U.S.A (1962) 48:1769-76. doi:10.1073/ pnas.48.10.1769

40. Steinberg MS. ON THE MECHANISM OF TISSUE RECONSTRUCTION BY DISSOCIATED CELLS, I. POPULATION KINETICS, DIFFERENTIAL ADHESIVENESS, AND THE ABSENCE OF DIRECTED MIGRATION. Proc Natl Acad Sci U.S.A (1962) 48:1577-82. doi:10.1073/pnas.48.9.1577

41. Steinberg MS. Reconstruction of Tissues by Dissociated Cells. Science (1963) 141:401-8. doi:10.1126/science.141.3579.401

42. Schötz EM, Burdine RD, Jülicher F, Steinberg MS, Heisenberg CP, and Foty RA. Quantitative Differences in Tissue Surface Tension Influence Zebrafish Germ Layer Positioning. HFSP J (2008) 2:42-56. doi:10.2976/1.2834817

43. Krens SFG, Veldhuis JH, Barone V, Čapek D, Maître JL, Brodland GW, et al. Interstitial Fluid Osmolarity Modulates the Action of Differential Tissue Surface Tension in Progenitor Cell Segregation during Gastrulation. Development (2017) 144:1798-806. doi:10.1242/dev.144964

44. Ninomiya H, David R, Damm EW, Fagotto F, Niessen CM, and Winklbauer R. Cadherin-dependent Differential Cell Adhesion in Xenopus Causes Cell 
Sorting In Vitro but Not in the Embryo. J Cel Sci (2012) 125:8. doi:10.1242/ jcs.095315

45. Pawlizak S, Fritsch AW, Grosser S, Ahrens D, Thalheim T, Riedel S, et al. Testing the Differential Adhesion Hypothesis across the EpithelialMesenchymal Transition. New J Phys (2015) 17:083049. doi:10.1088/13672630/17/8/083049

46. Bénazéraf B, and Pourquié O. Formation and Segmentation of the Vertebrate Body axis. Annu Rev Cel Dev Biol (2013) 29. doi:10.1146/annurev-cellbio101011-155703

47. Bénazéraf B, Francois P, Baker RE, Denans N, Little CD, and Pourquié O. A Random Cell Motility Gradient Downstream of FGF Controls Elongation of an Amniote Embryo. Nature (2010) 466:248-52. doi:10.1038/nature09151

48. Lawton AK, Nandi A, Stulberg MJ, Dray N, Sneddon MW, Pontius W, et al. Regulated Tissue Fluidity Steers Zebrafish Body Elongation. Development (2013) 140:573-82. doi:10.1242/dev.090381

49. Ochoa-Espinosa A, and Affolter M. Branching Morphogenesis: From Cells to Organs and Back. Cold Spring Harbor Perspect Biol (2012) 4. doi:10.1101/ cshperspect.a008243

50. Spurlin JW, Siedlik MJ, Nerger BA, Pang MF, Jayaraman S, Zhang R, et al. Mesenchymal Proteases and Tissue Fluidity Remodel the Extracellular Matrix during Airway Epithelial Branching in the Embryonic Avian Lung. Development (2019) 146. doi:10.1242/dev.175257

51. Varner VD, and Nelson CM. Cellular and Physical Mechanisms of Branching Morphogenesis. Development (2014) 141:2750-9. doi:10.1242/dev.104794

52. Jiang Y, Pjesivac-Grbovic J, Cantrell C, and Freyer JP. A Multiscale Model for Avascular Tumor Growth. Biophys J (2005) 89:3884-94. doi:10.1529/ biophysj.105.060640

53. Nath S, and Devi GR. Three-Dimensional Culture Systems in Cancer Research: Focus on Tumor Spheroid Model. Pharmacol Ther (2016) 163:94. doi:10.1016/ j.pharmthera.2016.03.013

54. Wallace DI, and Guo X. Properties of Tumor Spheroid Growth Exhibited by Simple Mathematical Models. Front Oncol (2013) 3. doi:10.3389/ fonc.2013.00051

55. Staneva R, Marjou FE, Barbazan J, Krndija D, Richon S, Clark AG, et al. Cancer Cells in the Tumor Core Exhibit Spatially Coordinated Migration Patterns. J Cel Sci (2019) 132. doi:10.1242/jcs.220277

56. Balkwill FR, Capasso M, and Hagemann T. The Tumor Microenvironment at a Glance. J Cel Sci (2012) 125:5591-6. doi:10.1242/jcs.116392

57. Mierke CT, Sauer F, Grosser S, Puder S, Fischer T, and Käs JA. The Two Faces of Enhanced Stroma: Stroma Acts as a Tumor Promoter and a Steric Obstacle. NMR Biomed (2018) 31:e3831. doi:10.1002/nbm.3831

58. Heine P, Lippoldt J, Reddy GA, Katira P, and Käs JA. Anomalous Cell Sorting Behavior in Mixed Monolayers Discloses Hidden System Complexities. New J Phys (2021) 23:043034. doi:10.1088/1367-2630/abf273

59. Elston CW, and Ellis IO. Pathological Prognostic Factors in Breast Cancer. I. The Value of Histological Grade in Breast Cancer: Experience from a Large Study with Long-Term Follow-Up. Histopathology (1991) 19:403-10.

60. Bazellières E, Conte V, Elosegui-Artola A, Serra-Picamal X, Bintanel-Morcillo M, Roca-Cusachs P, et al. Control of Cell-Cell Forces and Collective Cell Dynamics by the Intercellular Adhesome. Nat Cel Biol (2015) 17:409-20. doi:10.1038/ncb3135

61. Malinverno C, Corallino S, Giavazzi F, Bergert M, Li Q, Leoni M, et al. Endocytic Reawakening of Motility in Jammed Epithelia. Nat Mater (2017) 16: 587-96. doi:10.1038/nmat 4848

62. Merkel M, and Manning ML. A Geometrically Controlled Rigidity Transition in a Model for Confluent 3D Tissues. New J Phys (2018) 20:022002. doi:10.1088/1367-2630/aaaa13

63. Weigelin B, Bakker GJ, and Friedl P. Intravital Third Harmonic Generation Microscopy of Collective Melanoma Cell Invasion: Principles of Interface Guidance and Microvesicle Dynamics. Intravital (2012) 1:32-43. doi:10.4161/ intv. 21223

64. Thomas DG, Yenepalli A, Denais CM, Rape A, Beach JR, Wang Y, et al. Nonmuscle Myosin IIB Is Critical for Nuclear Translocation during 3D Invasion. J Cel Biol (2015) 210:583. doi:10.1083/jcb.201502039

65. Dahl KN, Kahn SM, Wilson KL, and Discher DE. The Nuclear Envelope Lamina Network Has Elasticity and a Compressibility Limit Suggestive of a Molecular Shock Absorber. J Cel Sci (2004) 117:4779-86. doi:10.1242/jcs.01357
66. Gerlitz G, and Bustin M. The Role of Chromatin Structure in Cell Migration. Trends Cel Biol (2011) 21:6-11. doi:10.1016/j.tcb.2010.09.002

67. Chow KH, Factor RE, and Ullman KS. The Nuclear Envelope Environment and its Cancer Connections. Nat Rev Cancer (2012) 12:196-209. doi:10.1038/ nrc3219

68. Harada T, Swift J, Irianto J, Shin JW, Spinler KR, Athirasala A, et al. Nuclear Lamin Stiffness Is a Barrier to 3D Migration, but Softness Can Limit Survival. J Cel Biol (2014) 204:669-82. doi:10.1083/jcb.201308029

69. Wolf $\mathrm{K}, \mathrm{Te}$ Lindert M, Krause M, Alexander S, Te Riet J, Willis AL, et al. Physical Limits of Cell Migration: Control by ECM Space and Nuclear Deformation and Tuning by Proteolysis and Traction Force. J Cel Biol (2013) 201:1069-84. doi:10.1083/jcb.201210152

70. Davidson PM, Denais C, Bakshi MC, and Lammerding J. Nuclear Deformability Constitutes a Rate-Limiting Step during Cell Migration in 3-D Environments. Cel. Mol Bioeng (2014) 7:293-306. doi:10.1007/s12195-014-0342-y

71. Fu Y, Chin LK, Bourouina T, Liu AQ, and VanDongen AMJ. Nuclear Deformation during Breast Cancer Cell Transmigration. Lab Chip (2012) 12:3774-8. doi:10.1039/C2LC40477J

72. Lomakin AJ, Cattin CJ, Cuvelier D, Alraies Z, Molina M, Nader GPF, et al. The Nucleus Acts as a Ruler Tailoring Cell Responses to Spatial Constraints. Science (2020) 370:eaba2894. doi:10.1126/science.aba2894

73. Venturini V, Pezzano F, Català Castro F, Häkkinen HM, Jiménez-Delgado $S$, Colomer-Rosell M, et al. The Nucleus Measures Shape Changes for Cellular Proprioception to Control Dynamic Cell Behavior. Science (2020) 370: eaba2644. doi:10.1126/science.aba2644

74. Palamidessi A, Malinverno C, Frittoli E, Corallino S, Barbieri E, Sigismund S, et al. Unjamming Overcomes Kinetic and Proliferation Arrest in Terminally Differentiated Cells and Promotes Collective Motility of Carcinoma. Nat Mater (2019) 18:1252-63. doi:10.1038/s41563-019-0425-1

75. Giavazzi F, Paoluzzi M, Macchi M, Bi D, Scita G, Manning ML, et al. Flocking Transitions in Confluent Tissues. Soft Matter (2018) 14:3471-7. doi:10.1039/ C8SM00126J

76. Serwane F, Mongera A, Rowghanian P, Kealhofer DA, Lucio AA, Hockenbery ZM, et al. In Vivo quantification of Spatially Varying Mechanical Properties in Developing Tissues. Nat Methods (2017) 14: 181-6. doi:10.1038/nmeth.4101

77. Nnetu KD, Knorr M, Käs J, and Zink M. The Impact of Jamming on Boundaries of Collectively Moving Weak-Interacting Cells. New J Phys (2012) 14:115012. doi:10.1088/1367-2630/14/11/115012

78. Devany J, Sussman DM, Yamamoto T, Manning ML, and Gardel ML. Cell Cycle-dependent Active Stress Drives Epithelia Remodeling. Proc Natl Acad Sci U.S.A (2021) 118:e1917853118. doi:10.1073/pnas.1917853118

79. Fritsch A, Höckel M, Kiessling T, Nnetu KD, Wetzel F, Zink M, et al. Are Biomechanical Changes Necessary for Tumour Progression? Nat Phys (2010) 6:730-2. doi:10.1038/nphys1800

80. Tambe DT, and Fredberg JJ. And I hope You like Jamming Too. New J Phys (2015) 17:091001. doi:10.1088/1367-2630/17/9/091001

81. Kim JH, Pegoraro AF, Das A, Koehler SA, Ujwary SA, Lan B, et al. Unjamming and Collective Migration in MCF10A Breast Cancer Cell Lines. Biochem Biophys Res Commun (2020) 521:706-15. doi:10.1016/ j.bbrc.2019.10.188

82. Etournay R, Popović M, Merkel M, Nandi A, Blasse C, Aigouy B, et al. Interplay of Cell Dynamics and Epithelial Tension during Morphogenesis of the Drosophila Pupal wing. Cambridge, United Kingdom: eLife Sciences Publications, Ltd (2015). doi:10.7554/eLife.07090

83. Friedl $\mathrm{P}$, and Mayor R. Tuning Collective Cell Migration by Cell-Cell Junction Regulation. Cold Spring Harbor Perspect Biol (2017) 9. doi:10.1101/ cshperspect.a029199

84. Kuan HS, Blackwell R, Hough LE, Glaser MA, and Betterton MD. Hysteresis, Reentrance, and Glassy Dynamics in Systems of Self-Propelled Rods. Phys Rev E (2015) 92:060501. doi:10.1103/PhysRevE.92.060501

85. Mongera A, Michaut A, Guillot C, Xiong F, and Pourquié O. Mechanics of Anteroposterior Axis Formation in Vertebrates. Annu Rev Cel Dev Biol (2019) 35:259-83. doi:10.1146/annurev-cellbio-100818-125436

86. Pillarisetti A, Desai JP, Ladjal H, Schiffmacher A, Ferreira A, and Keefer CL. Mechanical Phenotyping of Mouse Embryonic Stem Cells: Increase in Stiffness with Differentiation. Cell. Reprogramming (2011) 13:371-80. doi:10.1089/ cell.2011.0028 
87. Höckel M, and Behn U. The Order of Cancer: A Theory of Malignant Progression by Inverse Morphogenesis. Front Oncol (2019) 9. doi:10.3389/ fonc. 2019.00416

88. Kubitschke H, Wolf B, Morawetz E, Horn LC, Aktas B, Behn U, et al. Roadmap to Local Tumour Growth: Insights from Cervical Cancer. Scientific Rep 9 (2019) 1-14. doi:10.1038/s41598-019-49182-1

89. Seltmann K, Fritsch AW, Käs JA, and Magin TM. Keratins Significantly Contribute to Cell Stiffness and Impact Invasive Behavior. Proc Natl Acad Sci U.S.A (2013) 110:18507-12. doi:10.1073/pnas.1310493110

90. Siegel PM, and Massagué J. Cytostatic and Apoptotic Actions of TGF- $\beta$ in Homeostasis and Cancer. Nat Rev Cancer (2003) 3:807-20. doi:10.1038/ nrc1208

91. Höckel M. Morphogenetic fields of Embryonic Development in Locoregional Cancer Spread. Lancet Oncol (2015) 16:148-51. doi:10.1016/S1470-2045(14) 71028-9

92. Merkel M, Baumgarten K, Tighe BP, and Manning ML. A Minimal-Length Approach Unifies Rigidity in Underconstrained Materials. Proc Natl Acad Sci U.S.A (2019) 116:6560-8. doi:10.1073/pnas.1815436116

93. Petridou NI, Corominas-Murtra B, Heisenberg CP, and Hannezo E. Rigidity Percolation Uncovers a Structural Basis for Embryonic Tissue Phase Transitions. Cell (2021) 184:1914-28.e19. doi:10.1016/j.cell.2021.02.017

94. Doss BL, Pan M, Gupta M, Grenci G, Mège RM, Lim CT, et al. Cell Response to Substrate Rigidity Is Regulated by Active and Passive Cytoskeletal Stress. Proc Natl Acad Sci U.S.A (2020) 117:12817-25. doi:10.1073/pnas.1917555117
95. Gupta M, Doss B, Lim CT, Voituriez R, and Ladoux B. Single Cell Rigidity Sensing: A Complex Relationship between Focal Adhesion Dynamics and Large-Scale Actin Cytoskeleton Remodeling. Cell Adhes Migration (2016) 10: 554-67. doi:10.1080/19336918.2016.1173800

96. Kamkin A, and Kiseleva I. Mechanosensitivity In Cells And Tissues. Gent, Belgium: Academia (2005).

Conflict of Interest: The authors declare that the research was conducted in the absence of any commercial or financial relationships that could be construed as a potential conflict of interest.

Publisher's Note: All claims expressed in this article are solely those of the authors and do not necessarily represent those of their affiliated organizations, or those of the publisher, the editors and the reviewers. Any product that may be evaluated in this article, or claim that may be made by its manufacturer, is not guaranteed or endorsed by the publisher.

Copyright (c) 2021 Blauth, Kubitschke, Gottheil, Grosser and Käs. This is an openaccess article distributed under the terms of the Creative Commons Attribution License (CC BY). The use, distribution or reproduction in other forums is permitted, provided the original author(s) and the copyright owner(s) are credited and that the original publication in this journal is cited, in accordance with accepted academic practice. No use, distribution or reproduction is permitted which does not comply with these terms. 